\title{
Building on the RCP FallSafe care bundles: Is observation and review the key to reducing inpatient falls? The Northumbria experience 2013-2020 沙
}

\author{
Authors: David A Richardson, ${ }^{A}$ David Dawson ${ }^{B}$ and Elaine Henderson ${ }^{C}$
}

The Royal College of Physicians (RCP) FallSafe care bundles are the recommended foundation for inpatient falls prevention in the UK. Yet there is a paucity of data to support its widespread use and the reductions in falls demonstrated in the original pilot and in subsequent small studies have not yet been reproduced in larger patient groups. Northumbria Healthcare NHS Foundation Trust (NHCFT) has seen a significant reduction in falls, falls per 1,000 bed days and harm from falls between 2013-2020 when combining the RCP FallSafe care bundles with a supportive observation policy (SOP) related to falls prevention. Highlighting the potential cost savings ( $£ 5.3$ million over a 3 -year period) has supported the growth and development of the NHCFT inpatient falls prevention service and the implementation of the SOP.

KEYWORDS: RCP FallSafe care bundles, inpatient falls, supportive observation

DOI: $10.7861 /$ clinmed.2020-0482

\section{Introduction}

Around 250,000 patient falls are reported from acute, community and mental health units in England and Wales annually., They can cause serious injury and death and are estimated to cost hospitals $£ 630$ million annually. ${ }^{2}$ Systematic review suggests a multifactorial assessment and intervention such as the Royal College of Physicians (RCP) FallSafe care bundles can reduce falls by $20-30 \% .^{3,4}$ To date, however, there is little evidence to show that widespread implementation of these measures of good practice has occurred and that they can effect such an improvement. ${ }^{5}$

Authors: ${ }^{\text {A }}$ consultant physician and geriatrician and falls clinical lead, Northumbria Healthcare NHS Foundation Trust, North Shields, UK; ${ }^{B}$ King's College older persons nurse fellow, falls and frailty matron and falls strategic lead, Northumbria Healthcare NHS Foundation Trust, North Shields, UK; ' director of nursing, Northumbria Healthcare NHS Foundation Trust, North Shields, UK
Northumbria Healthcare NHS Foundation Trust (NHCFT) includes one specialist emergency care hospital, three teaching district general hospitals and four community hospitals. There are 37 wards of varying age, design layout and specialty (including mental health), with a total of 999 beds. We report the experience of the NHCFT falls team with the RCP FallSafe care bundles (building on our initial experience in 2013) and the effects on inpatient falls outcome measures following an increased focus on the post inpatient fall RCP Falls care bundle and the introduction of a supportive observation policy (SOP) relating to fall prevention in $2017 .^{6}$

\section{Methods}

As previously reported, the RCP FallSafe care bundles were modified in 2013 and some measures adapted to ensure a trust-wide ability to comply and to allow for easier audit. ${ }^{6}$ The results of subsequent national and local NHCFT audits are shown in Table 1. Further modifications, to take account of the latest National Institute for Health and Care Excellence (NICE) guidelines and technological advances, are outlined in Table 2. ${ }^{9,10}$ In order to improve compliance, when a patient is admitted nurses complete a falls care plan (FCP) which is based on the RCP FallSafe care bundles, as part of the electronic nursing admission documentation (using NerveCentre software).

In 2017, due to expansion of the nursing team, the NHCFT falls team were able to start undertaking more thorough reviews following inpatient falls in addition to their usual clinical, advisory and teaching workload. When an incident record (IR1) for a fall is completed on Datix, a copy and an alert is automatically sent to the falls team email inbox. The falls team then review the IR1 and the electronic patient record; this includes physiological observations, nursing records, FCP and level of observation (recorded using NerveCentre software), patient investigations (recorded using ICE software), the patient's medication (recorded using MedChart) and any patient radiology (recorded using VueMotion). This is then followed up by a ward visit where possible and an entry written in the medical records summarising and alerting the ward team to any issues with compliance with the Falls Care bundles, potential fall risk factors, level of observation and recommendations for invention if required. The FCP is then amended in NerveCentre and a falls alert is added to MedChart and the patient administration system (PAS). The data are then prospectively recorded in an MS Excel database for audit purposes. 
Table 1. Compliance with FallSafe care bundle for all patients 2013-2020, \%

$\begin{array}{lllllll}\text { Richardson } & \text { RCP national } & \text { Local } & \text { Post-fall } & \text { RCP national } & \text { Local } & \text { Data } \\ \text { et al } 2013^{5} & \begin{array}{l}\text { audit of } \\ \text { inpatient falls }\end{array} & \begin{array}{l}10 \text {-ward } \\ \text { data }\end{array} & \begin{array}{l}\text { review } \\ \text { data }\end{array} & \begin{array}{l}\text { audit of } \\ \text { inpatient falls }\end{array} & \begin{array}{l}\text { 37-ward } \\ \text { data }\end{array} & \begin{array}{l}\text { collected } \\ \text { for CQUIN }\end{array} \\ & 2015^{7 a} & 2017^{b} & 2017^{c} & 2017^{5} & 2018^{\text {d }} & \text { 2019/2020 } \\ & & & & & & \text { action CCG7 }\end{array}$

\section{Bundle for all patients}

A history of previous falls and of

fear of falling is taken at the time

of admission

Urinalysis is conducted on

admission

New prescriptions of night sedation are avoided

A call bell is in reach

Appropriate footwear is available and in use

There is assessment for and provision of walking aids within 24 hours

Bundle for older and more vulnerable patients

A cognitive assessment is

conducted in all admissions aged

$>70$ years

Those at risk are tested for

delirium

An assessment of risk versus

benefit for use of a bedrail is

conducted

Visual assessment is conducted $\quad 84$

Lying and standing blood pressure

Medication is reviewed with respect

to cardiovascular and central

nervous system acting medications

Based on observation, toileting arrangements are assessed and planned
97

84

84

99

86,97

84

99

92
28,79
32,39

32,59

58,63
89
82

98

54

96

89

98

$97 \quad 95 \quad 62$

62

96

87

$94 \quad 85 \quad 91 \quad 97$

$58-69 \quad 55 \quad 41$

98

99

$\begin{array}{llll}94 & 89 & 97 & 96 \\ 82 & 78 & 35 & 70 \\ 90 & 92 & 68 & 97\end{array}$

85

${ }^{a}=$ data for two hospital sites; ${ }^{b}=$ audit of FallSafe care bundles on 10 wards, May 2016 to March 2017: all patients over 65 years in hospital over 12 hours:

${ }^{c}=$ post-inpatient-fall audit, 2017, $n=99 ;^{d}=$ audit of FallSafe care bundles on 37 wards, Oct 2018 to November 2018: all patients over 65 years in hospital over

12 hours; CQUIN $=$ Commissioning for Quality and Innovation; RCP $=$ Royal College of Physicians.

Similarly, when a patient who has had a previous inpatient fall is re-admitted to hospital, an alert is sent to the falls team by PAS, so they can ensure the appropriate FCP and level of observation are in place at the start of any subsequent admission.

An SOP related to falls prevention was introduced in January 2017 which had four levels of nursing observation for falls prevention: routine care rounding (every 2 hours), increased care rounding (every 15-60 minutes), 'line of sight' and 'within arm's length' (supplementary material S1). As part of the FCP, nurses assign and record one of these levels of observation based on their clinical judgement. The level is displayed on the electronic live ward patient summary screens, allowing both staff and management to know how many patients on a ward require a specific level of observation at any one time. This information can be updated if the clinical situation changes and accessed remotely and in real time, which allows for the movement or provision of additional staff to ward areas within the trust where there are a high prevalence of patients requiring more close observation. To support nursing staff in setting the correct level of observation, the avoiding falls level of observation assessment tool (AFLOAT) was developed as part of our involvement in the NHS Improvement Falls Collaborative 2018. This tool (supplementary material S2) 
Table 2. RCP FallSafe care bundle measures in Northumbria Healthcare NHS Foundation Trust

RCP FallSafe care bundle measure

\section{Bundle for all patients}

A history of previous falls and of fear of falling is taken at the time of admission

Urinalysis is conducted on admission

New prescriptions of night sedation are avoided

A call bell is in reach

Appropriate footwear is available and in use

There is immediate assessment for and provision of walking aids

\section{Bundle for older and more vulnerable patients}

A cognitive assessment (Montreal cognitive assessment (MoCA) or abbreviated mental test score (AMTS)) is conducted in all admissions aged $>70$ years

Those at risk are tested for delirium (confusion assessment method (CAM))

An assessment of risk versus benefit for use of a bedrail is conducted

Visual assessment is conducted

Lying and standing blood pressure are taken with a manual sphygmomanometer

Medication is reviewed with respect to cardiovascular and central nervous system acting medications

\section{NHCFT adaptation/method of data collection}

Recorded in the adult nursing documentation (electronic software NerveCentre). A positive answer to this question then directs the nursing team to complete the falls care plan in NerveCentre. This measure can be audited remotely

Urinalysis has now been removed from the RCP FallSafe care bundle in line with NHSI CQUIN 2019/2020

No prescriptions of night sedation. NHCFT now uses the electronic software MedChart for all inpatient medication prescription. All medications listed as a hypnotic or sedative in the BNF have an alert, so that if prescribed on admission or during the inpatient stay, the prescriber is alerted to the fact that this medication is a falls risk factor and that they need to identify a rationale for its new prescription or its continuation, before it can be prescribed in hospital in compliance with NHSI CQUIN 2019/2020. This measure can be audited remotely

Measure usually audited at the patient's bed space. Some patients, who were freely ambulant, were not included when using day rooms or sitting areas. However, immobile patients, or patients who required assistance to mobilise, who were placed in such areas are audited

A significant number of elderly patients are admitted without suitable or appropriate footwear. For those without appropriate footwear, the trust has purchased supplies of slipperettes. These come in three sizes and have a sole with rubber grips, which, if worn appropriately, make mobilising on the shiny hospital floors less hazardous. In addition, for those who require slippers but cannot source them, a limited supply is available after a physiotherapy review

Measure audited at the patient's bed space. We initially found there was a misplaced perception that by taking the walking aid away from a confused patient you will stop them attempting to mobilise. This had led to the walking aids being tidied at the end of the bay or deliberately placed away from the patient's immediate reach. Where this problem was encountered additional education was provided to the ward and therapy staff by the falls team

Assessed by reviewing the information recorded in the ED admission document and the adult nursing documentation (electronic software NerveCentre) part of an existing trust CQUIN project

Assessed by reviewing the information recorded in the ED admission document and the adult nursing documentation (electronic software NerveCentre) - part of an existing trust CQUIN project

Assessed by reviewing the 'bed height, rail and mat matrix' included in the adult nursing documentation (electronic software NerveCentre)

Spectacles are within sight and reach: this measure was added into the care bundle for all patients and audited at the patient's bed space

Visual assessment: the first two parts of the RCP visual assessment are recorded in the adult nursing documentation (electronic software NerveCentre)

Recorded using automated blood pressure monitoring devices as per a specific protocol included in the adult nursing documentation (electronic software NerveCentre). Additional training and support for ward teams is provided from the NHCFT falls team

Assessed by review of the patient's drug charts (electronic software MedChart) and comparing the medication list to Medicines and falls in hospital: guidance sheet ${ }^{11}$ 
Table 2. RCP FallSafe care bundle measures in Northumbria Healthcare NHS Foundation Trust (Continued)

Based on observation, toileting arrangements are assessed and planned (tailored to needs rather than the standard 2-hourly arrangement)

\section{Bundle for after a fall}

After a fall, appropriate assessments and procedures are followed, including neurological observations in those who have hit their head or had an unwitnessed fall

A post-fall review (how can further falls be prevented for this patient) is conducted
Assessed by reviewing the continence pathway included in the adult nursing documentation (electronic software NerveCentre)

The falls team had previously modified a Nottingham University Hospitals template for a medical report following a fall and produced a sticker, which is inserted into the medical notes and is completed by the doctor or nurse practitioner after reviewing the patient who has fallen

This sticker and the medical and nursing responses to the fall, are audited by members of the NHCFT falls team against the measures recommended

BNF = British National Formulary; ED = emergency department; CQUIN = Commissioning for Quality and Innovation; NHCFT = Northumbria Healthcare NHS Foundation Trust; NHSI = NHS Improvement; RCP = Royal College of Physicians.

assigns points depending on features of clinical presentation (such as previous falls, confusion, postural hypotension), with the total score corresponding to one of the four levels above.

\section{Outcome data}

Data for falls and occupied bed days were collected from the trust incident reporting system and occupancy data and falls per 1,000 bed days could be calculated. When reporting an injury, the National Patients Safety Agency (NPSA) classifications of harm were used. ${ }^{2}$

\section{Results}

During this period 2013-2020, there was no obvious change in overall compliance with the first two RCP FallSafe care bundles (Table 1). Following more focused work on the third RCP FallSafe care bundles and the introduction of the SOP in 2017, there has been a significant reduction in falls per 1,000 bed days (Fig 1). There was also a reduction in the total number of patients who suffered an inpatient fall and in those patients with recurrent

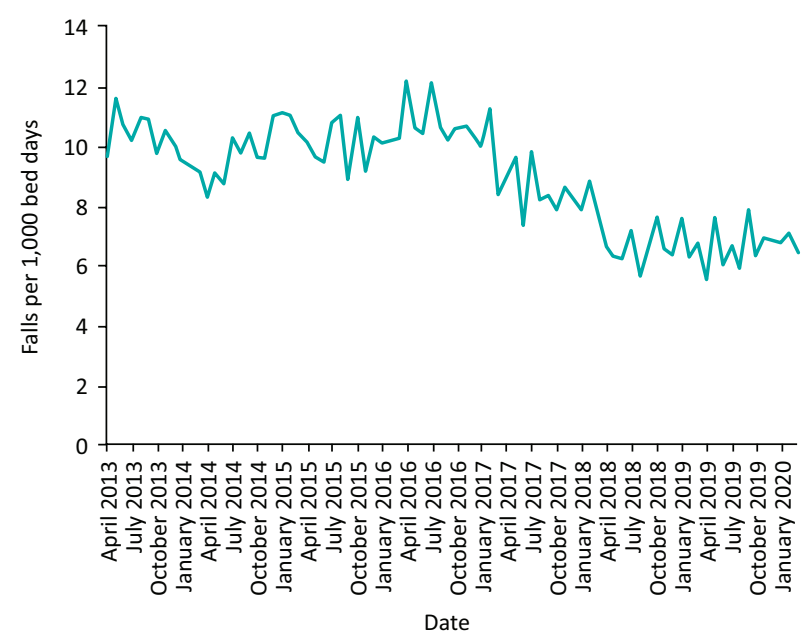

Fig 1. Inpatient falls per 1,000 bed days at Northumbria Healthcare NHS Foundation Trust 2013-2020.

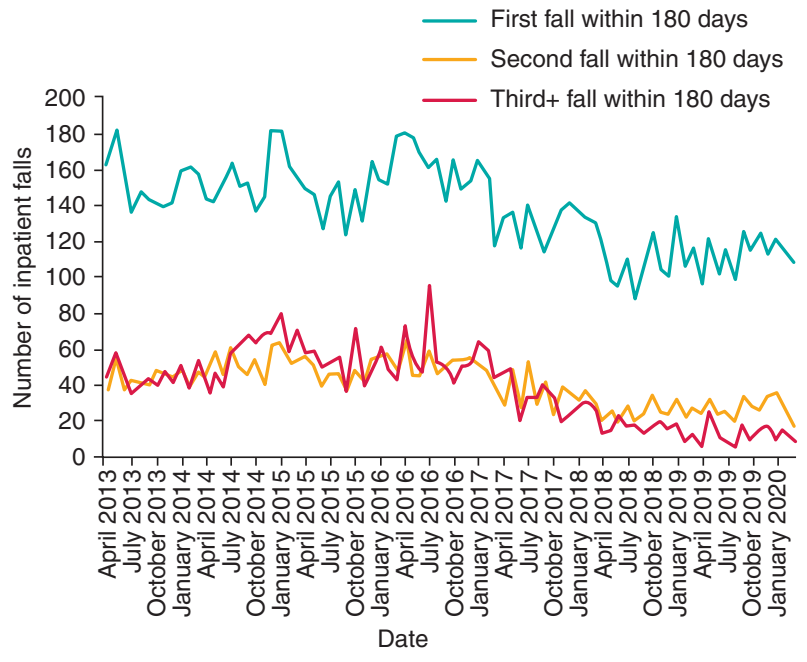

Fig 2. Number of inpatients with falls and subsequent falls at Northumbria Healthcare NHS Foundation Trust 2013-2020.

inpatient falls (Fig 2). During the period 2013-2020 the number of inpatient falls with harm declined (Fig 3) and the incidence of inpatient hip fractures has halved (Fig 4).

\section{Discussion}

Following an increased focus on the post inpatient fall RCP FallSafe care bundle and the introduction of a SOP relating to fall prevention in 2017, NHCFT has seen a significant reduction in falls, falls per 1,000 bed days, falls with harm and inpatient hip fractures. This has potentially saved NHCFT $£ 5.3$ million, of which $€ 383,000$ is due to the reduction in inpatient hip fractures, compared to the estimated costs prior to $2017 .^{2}$ It has also potentially saved over 17,000 bed days by reducing length of stay, of which 600 is due to the reduction in inpatient hip fractures. One of the most important aspects of the post inpatient RCP FallsSafe care bundle is that it seeks to identify, and address if possible, factors that led to the inpatient fall in that patient and prevent them having further inpatient falls. Many possible factors need to be considered and the investigator needs to 


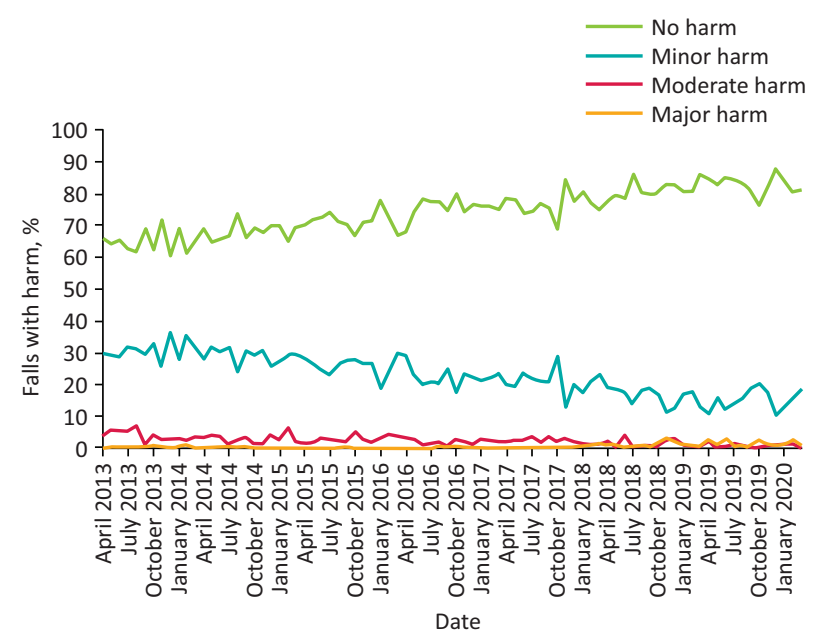

Fig 3. Number of inpatient falls with harm at Northumbria Healthcare NHS Foundation Trust 2013-2020.

address not only patient factors, but also environmental and often organisational factors. In NHCFT, this increased focus on the post-inpatient fall reviews has been made possible by additional recruitment into the NHCFT falls team. This now allows all IR1s relating to falls to be reviewed by the falls team. Recommendations made regarding the FallSafe care bundle measures and the further management of specific falls risk factors identified (eg medication changes, the use of compression hosiery, etc) are recorded in the medical records and discussed with the ward nursing and medical team.

The NHCFT falls team recognised from our weekly IR1 review meetings and serious incident (SI) reports that some patients who fell and came to harm had not had the appropriate level of observation set or carried out. Lack of robust assessment, documentation, or rationale for setting a level of observation all contributed to suboptimal care. The Crown Court case of the Crown v Shrewsbury and Telford NHS Trust highlighted the national legal narrative on inpatient fall prevention. ${ }^{12}$ The deaths of five patients following falls were investigated. A recurring theme was the lack of observation of the patients and the lack of 'enhanced care'. NHCFT received a similar narrative following two local coroners' inquiries. The coroner's findings included comments about the lack of clarity relating to the level of observation the patient should have received at the time they fell.

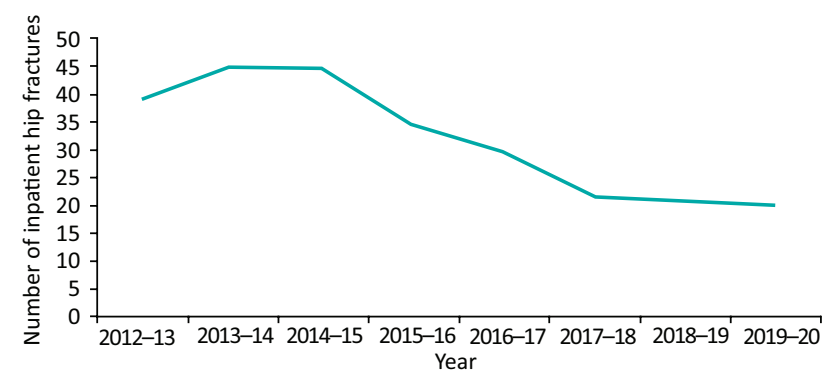

Fig 4. Incidence of inpatient hip fractures at Northumbria Healthcare NHS Foundation Trust 2013-2020.
In response to these local and national drivers, we launched an SOP relating to falls and subsequently developed AFLOAT, as detailed earlier. Feedback on the use of the SOP and AFLOAT has been positive from staff, patients and their relatives.

The SOP relating to falls has been subject to criticism and scrutiny within NHCFT due to the perceived additional costs of staffing needed at times to provide the correct level of observation. Using the NHS Improvement modelling, the increased costs of staffing has been shown to be more than offset by the overall savings to the organisation. Trust board recognition of this has been crucial to ensure that both the falls team and SOP are adequately resourced and sustainable going forward.

There may be other factors, not readily apparent, that may have contributed to the reduction in inpatient falls seen from 2013 to 2020. The increased profile of the NHCFT falls team on the wards, along with regular FallSafe care bundle audit and promotional activities, may have heightened awareness of the inpatient falls strategy throughout the trust. The NHCFT reconfiguration in 2015 to a single point of access specialist emergency care hospital, an overall reduction in LOS and a reduction the number of care of the elderly and community hospital beds may all have had some bearing.

In summary, when properly resourced the RCP FallSafe care bundles, used in combination with a robust SOP relating to falls, can lead to significant improvements in inpatient fall and inpatient hip fracture reduction. Trust board members and clinical commissioning groups should consider investing in their local inpatient falls prevention services to reduce patient harm and to save overall costs.

\section{Supplementary material}

Additional supplementary material may be found in the online version of this article at www.rcpjournals.org/clinmedicine: S1 - Northumbria Healthcare supportive observation policy for falls.

S2 - Avoiding falls levels of observation assessment tool (AFLOAT).

\section{Acknowledgements}

We would like to acknowledge the other members of the NHFCT falls team who contributed significantly to this quality improvement work: $\mathrm{Dr}$ Ruth Ross, consultant physician/geriatrician; Dr Mira Doshi, consultant physician/geriatrician; Philippa Whitelaw, falls nurse practitioner; Shona Stafford-Johnston, falls nurse practitioner; Gary Maltby, falls nurse practitioner; Doreen Hendrie, falls nurse; Joanne Turnbull, falls nurse; and Angela McArdle, falls administration support officer.

\section{References}

1 National Patient Safety Agency. Slips trips and falls in hospital. NPSA, 2007.

2 NHS Improvement. The incidence and costs of inpatient falls in hospitals. NHSI, 2017. https://improvement.nhs.uk/resources/ incidence-and-costs-inpatient-falls-hospitals

3 Royal College of Physicians. FallSafe care bundles. RCP, 2011. www. rcplondon.ac.uk/guidelines-policy/fallsafe-resources-original

4 Health Foundation. Closing the gap through clinical communities: The FallSafe project. Health Foundation, 2015. www.rcplondon. ac.uk/projects/outputs/health-foundation-fallsafe-report

5 Royal College of Physicians. National audit of inpatient falls: Audit report 2017. RCP, 2017. www.rcplondon.ac.uk/projects/outputs/ naif-audit-report-2017 
6 Richardson DA, Bhagwat A, Forster K et al. The Royal College of Physicians' Fallsafe care bundles applied trustwide; the Northumbria experience 2013. Clin Med 2015;15:530-5.

7 Royal College of Physicians. National audit of inpatient falls: Audit report 2015. RCP, 2015. www.rcplondon.ac.uk/projects/outputs/ naif-audit-report-2015

8 NHS England. Commissioning for Quality and Innovation (CQUIN): guidance for 2019-20. NHSE, 2019. www.england.nhs.uk/publication/ commissioning-for-quality-and-innovation-cquin-guidancefor-2019-2020

9 National Institute of Health and Care Excellence. Falls in older people: assessing risk and prevention. Clinical guideline CG161. NICE, 2013. www.nice.org.uk/guidance/cg161.

10 National Institute for Health and Care Excellence. Falls in older people: Quality Standard [QS86]. NICE, 2017. www.nice.org.uk/ guidance/qs86
11 Royal College of Physicians. Medicines and falls in hospital: guidance sheet. RCP, 2011. www.rcplondon.ac.uk/guidelines-policy/fallsaferesources-original

12 Judiciary of England and Wales. Regina (on prosecution by the Health and Safety Executive) $v$ Shrewsbury and Telford NHS Trust: sentencing remarks of the Hon. Mr Justice Haddon-Cave at Stafford Crown Court, 28th November 2017. Judiciary of England and Wales, 2017. www.judiciary.uk/wp-content/uploads/2017/11/ r-v-shrewsbury-nhs-judgment-1.pdf

Address for correspondence: Dr David A Richardson, Wansbeck General Hospital, Woodhorn Lane, Ashington, Northumberland NE63 9J], UK.

Email: david.richardson@northumbria-healthcare.nhs.uk

Peer support can help staff cope with the stress that comes from their work. New guidance from the Royal College of Physicians and Royal College of Psychiatrists sets out the values, principles and practice of peer support.

Download the guidance and accompanying infographic: www.rcplondon.ac.uk/projects/peer-support-guidance
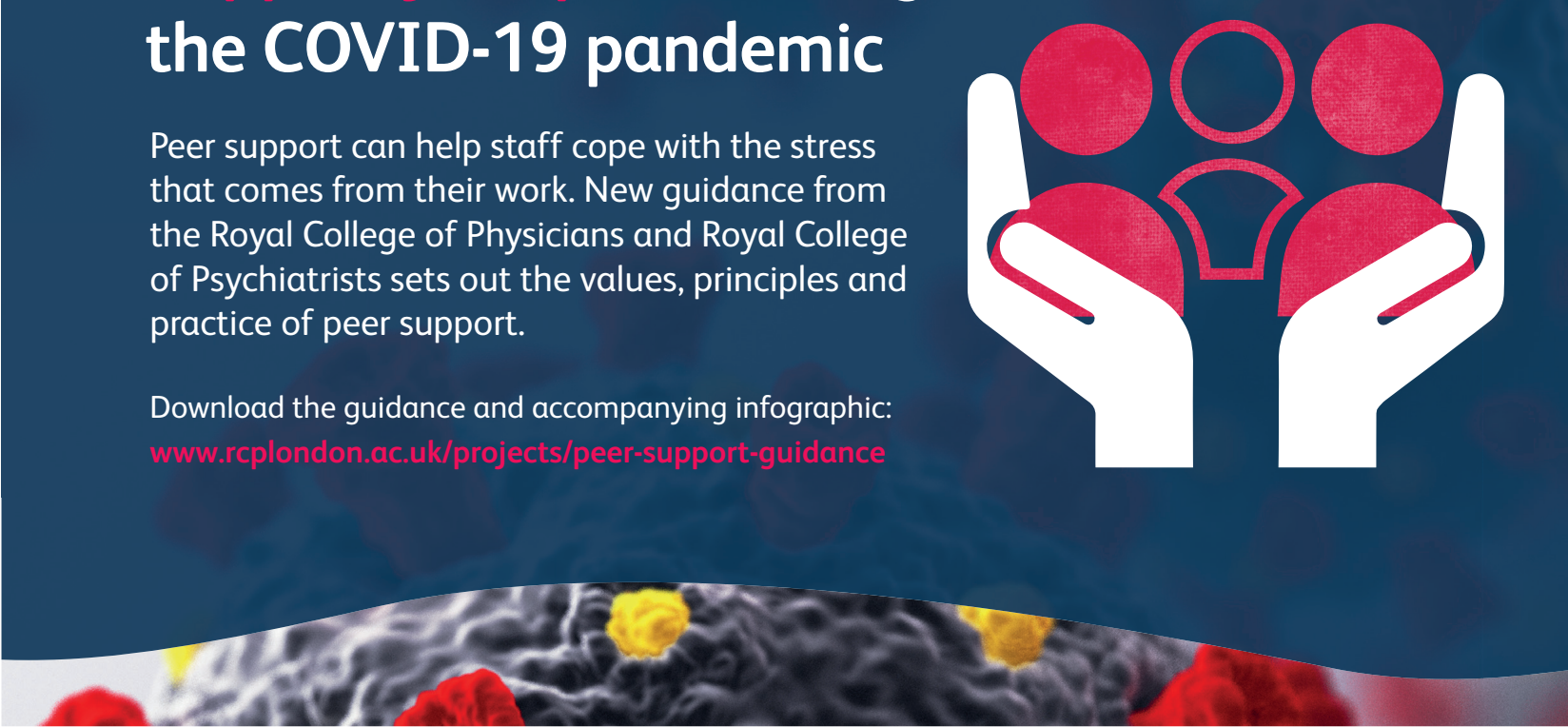